\title{
Potencialidades computacionales del Método de los Elementos Finitos para la modelación y simulación de materiales compuestos: revisión
}

\author{
Computational potentialities of the Finite Elements \\ Method for the Modeling and Simulation \\ of Composite Materials: A review
}

Ismeli Alfonso ${ }^{1}$, Vladimir Rodríguez-Iglesias ${ }^{2}$, Ignacio Alejandro Figueroa $^{1}$

\footnotetext{
${ }^{1}$ Instituto de Investigaciones en Materiales. Universidad Nacional Autónoma de México. Circuito Exterior SN. Ciudad Universitaria. CP. 04510. Del. Coyoacán, México, DF. México. Tel: +52-55-56232857. e-mail: ialfonso@unam.mx; iafigueroa@unam.mx

${ }^{2}$ Facultad de Ingeniería. Universidad Autónoma del Carmen. Campus III. Avenida Central S/N, Esq. con Fracc. Mundo Maya, C.P. 24115, Ciudad del Carmen, Campeche, México.

e-mail: viglesias@gmail.com
}

\section{RESUMEN}

En este trabajo se presenta una revisión bibliográfica de modelos computacionales que utilizan el Método de los Elementos Finitos (MEF) para el estudio del comportamiento mecánico de los materiales compuestos, enfocándose el análisis en materiales reforzados por partículas o fibras. El análisis realizado muestra que el MEF permite estimar, de manera efectiva, la influencia sobre las propiedades mecánicas de los materiales compuestos, de factores como el reforzante, su fracción volumétrica, orientación, relación de aspecto y tamaño. Esta revisión mostró que la propiedad más estudiada es el módulo de Young, así como los modelos que favorecen la disminución de la geometría utilizando las ventajas computacionales. Se destaca el trabajo futuro que requiere atención.

Palabras clave: Método de los Elementos Finitos, MEF, compuestos, reforzantes, modelación, simulación.

\section{ABSTRACT}

This work presents a review of computational models that use the Finite Elements Method (FEM) for the study of composite materials mechanical behavior, focusing the analysis in materials reinforced by particles or fibers. Bibliographic analysis shows that using FEM it is possible to successfully estimate the effect on the composites mechanical properties, of parameters such as the reinforcement type, volumetric fraction, orientation, aspect ratio and size. This review showed that the most studied property is Young's modulus, as well as the models that favor the geometry decrease using the computational advantages. Future work that needs attention is addressed.

Keywords: Finite Elements Method, FEM, composites, reinforcement, modeling, simulation.

\section{INTRODUCCIÓN AL MÉTODO DE LOS ELEMENTOS FINITOS}

En la actualidad, es de gran importancia el estudio de los materiales, así como de los sistemas o estructuras en los cuales operan estos materiales. En muchas ocasiones, antes de fabricar un material o un prototipo, es deseable contar con información que permita tomar decisiones sobre el proceso final de fabricación. Por tal motivo, se ha incrementado de manera notable el uso de la modelación y la simulación. El Método de los Elementos Finitos (MEF) constituye hoy en día un procedimiento habitual de modelación y simulación, tanto en mecánica estructural como en mecánica de sólidos. Su uso se ha extendido también a la resolución de problemas de transferencia de calor, mecánica de fluidos y electromagnetismo [1]]. El nacimiento y desarrollo del MEF es una consecuencia de la disponibilidad de herramientas electrónicas de cálculo cada vez más potentes desde finales del siglo XX. En el MEF las estructuras se dividen en zonas o elementos conectados me- 
diante nodos, pero a diferencia del cálculo matricial, dentro de ellos solo se conoce la solución de manera aproximada en función de los movimientos nodales, que definen la solución dentro de cada uno de los elementos en que se divide la estructura y, por ende, la solución en toda ella [2].

El primer trabajo sobre este tema data de 1956, publicado por Turner y col. [3]. No obstante desde los antiguos egipcios podemos encontrar vestigios de este tipo de cálculos para determinar el volumen de las pirámides. Arquímedes (287-212 a.C.) empleaba el mismo método para calcular áreas y volúmenes diversos. El MEF nació como una herramienta ingenieril, y su desarrollo ha estado siempre muy vinculado a la presión de la industria por resolver problemas concretos. Incluso el primer libro importante en que se analiza el MEF desde el punto de vista matemático se publicó en 1973, cuando el método llevaba al menos quince años empleándose en la industria [4]. Su uso se extendió hacia finales de los sesenta con la aparición de los primeros programas comerciales. En las décadas de los setenta y ochenta ocurrió un auge en el uso del MEF gracias a la revolución computacional.

El MEF se ha integrado a la ingeniería y al diseño asistidos por computadora con la finalidad de reducir los costos y tiempos de proyecto o de puesta de productos en el mercado [2]. En el área de Ciencia e Ingeniería de Materiales, el MEF se ha venido usando de manera importante para el análisis de materiales avanzados, como es el caso de los materiales compuestos, los nanotubos de carbono y los materiales porosos, entre otros [ㅁ-ㅁ]. También se ha utilizado para el estudio de procesos tales como la fundición $[\underline{10}, \underline{11}]$ y la soldadura [12], y fenómenos como la corrosión [13] y el desgaste [14]. En el caso de análisis estructural, el MEF divide la estructura en elementos, cada uno de los cuales presenta su propia matriz de rigidez. En este caso la ecuación fundamental que gobierna el fenómeno es la determinación del módulo de Young mediante la relación esfuerzo-deformación.

\section{PROGRAMAS DE CÓMPUTO QUE EMPLEAN EL MEF}

En la actualidad existen diferentes programas de análisis por elementos finitos, entre los cuales están ABAQUS, ANSYS, COSMOS, PATRAN, NASTRAN, ALGOR, STRUDL y CAEPIPE, MARC and ALTAIR HYPERWORKS. Estos programas contienen herramientas que permiten el modelado y la simulación de un gran número de fenómenos, estructuras y materiales, para lo cual divide su operación en etapas o módulos, donde generalmente se definen aspectos tales como geometría del modelo, tipos de elementos en los que se dividirá, asignación de propiedades de los materiales y generación de la malla para definir los nodos y uniones entre elementos. Después se asignan las condiciones a las que está sometido el modelo, tales como fuerzas, presiones, restricciones de movimiento y temperatura; y por último se realiza un análisis de los resultados obtenidos para los nodos y/o elementos y para el modelo en general, obteniéndose datos mediante tablas y gráficos.

De acuerdo al tipo de geometría y las condiciones de carga a las que está sometido un material, se pueden utilizar diferentes tipos de elementos, cuya unión modela la totalidad de la pieza, material o estructura. En la Figura 1 se pueden observar algunos tipos de elementos con que cuenta el ANSYS 15.0 M.R. [15], uno de los programas anteriormente mencionados que utilizan el MEF. Cada uno de estos elementos tiene diferentes características, entre las cuales están grados de libertad, especificación de análisis bi o tridimensionales, cantidad de nodos en el mallado, variables de entrada que se necesitan agregar al modelo, y datos de salida.
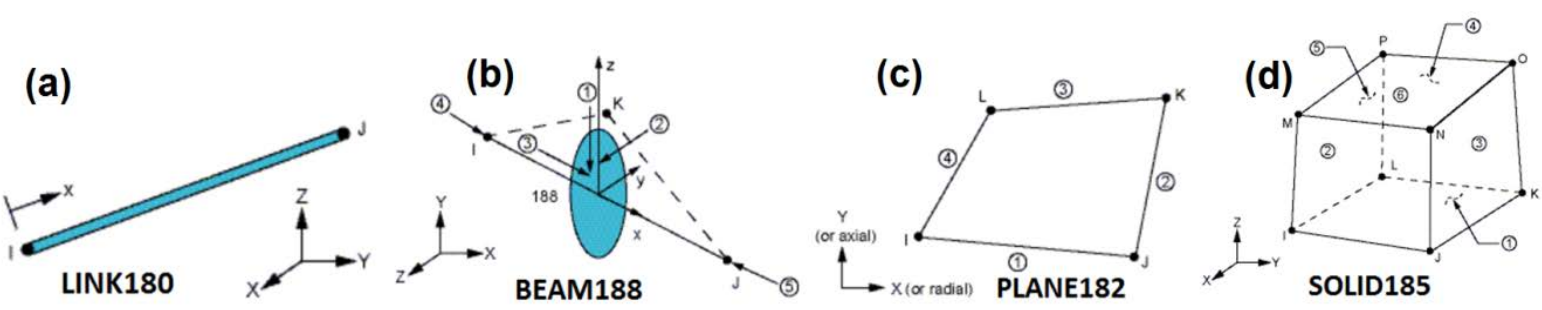

Figura 1: Algunos elementos con que cuenta el ANSYS 15.0: (a) LINK8, (b) BEAM188, (c) PLANE182, y (d) SOLID185. Modificado de [토] .

Con el apoyo computacional se pueden resolver problemas muy complejos como es el caso de la determinación del módulo de elasticidad de nanotubos de carbono con elementos tan sencillos como vigas (beam), o de estructuras o materiales tridimensionales con el uso de elementos sólidos (solid), como se analizará más adelante. 


\section{LOS MATERIALES COMPUESTOS}

Los materiales compuestos o compósitos se definen como una combinación de dos o más materiales diferentes, con una interfase reconocible entre ellos. También pueden definirse como aquellos materiales que contienen un reforzante soportado por una matriz [16]. Sus propiedades mecánicas y térmicas superiores, y su baja densidad los hacen muy atractivos [17]. El reforzante puede presentar forma de partículas, fibras o láminas, y es quien comanda las propiedades del compósito, ya que algunas de sus propiedades pueden llegar a ser entre 20 y 100 veces superiores a las de la matriz [18]. En cuanto a las matrices, su propósito es soportar al reforzante, protegerlo del ambiente y transferir carga. Según el tipo de matriz se dividen en poliméricos, cerámicos y metálicos [16]. Entre las características más importantes que definen a los materiales compuestos está la fracción volumétrica del reforzante, muy utilizada para determinar sus propiedades macroscópicas.

Debido a la gran variedad de formas de los reforzantes, se debe prestar atención especial a este detalle, pues en casos como en que los reforzantes tienen una relación de aspecto (largo/ancho) baja la transferencia de carga no es tan eficiente como cuando el reforzante es una fibra continua [19]. Incluso partículas con relaciones de aspecto iguales pueden tener formas tan diferentes como esferas, elipses, cubos o irregulares, como lo describen Kumar y colaboradores [20]. Por tal motivo, algunas variables claves para una correcta determinación de las propiedades de los materiales compuestos son la fracción volumétrica y la forma del reforzante, lo cual se incluye en varios modelos teóricos. El más sencillo de estos modelos es la regla de las mezclas, la cual establece que determinada propiedad de un material compuesto depende de la fracción volumétrica del reforzante y del valor de dicha propiedad para el reforzante y la matriz. No obstante esta regla no toma en cuenta algunos aspectos fundamentales, como por ejemplo la relación de aspecto de los reforzantes y su orientación, lo cual sí es incluido en otros modelos, estando entre.

- los más utilizados los de Halpin-Tsai [21], de Hashin y Shtrikman [22], Eshelby [23], Mura [24], Kroner y Budiansky [25, 26], Guth [27], así como el de Lewis y Nielsen [28]. Debido a la importancia que se le ha dado en los trabajos encontrados en la bibliografía, a continuación se destaca el modelo de Halpin-Tsai, para el cual el módulo de Young se puede determinar mediante la relación:

$$
E_{c}=\frac{E_{m}\left(1+2 s q V_{p}\right)}{1-q V_{p}}
$$

donde $E_{c}, E_{p}$ y $E_{m}$ son respectivamente los módulos de Young para el compuesto, el reforzante y la matriz; $s$ es la relación de aspecto del reforzante; $V_{p}$ su fracción volumétrica; y $q$ es un parámetro geométrico que depende en parte de $s$. Este modelo ha probado tener una alta eficiencia y es muy utilizado. El MEF se ha sumado a estos modelos como una importante herramienta para estimar el comportamiento de los compósitos, fundamentalmente debido a la disponibilidad de herramientas electrónicas de cálculo cada vez más potentes.

\section{MODELOS REPORTADOS PARA LA ESTIMACIÓN DEL COMPORTAMIENTO MECÁNICO DE MATE- RIALES COMPUESTOS MEDIANTE EL MEF}

Se han encontrado un gran número de trabajos que utilizan el método de los elementos finitos para estimar las propiedades de los materiales compuestos. El MEF es una herramienta muy útil para el estudio de los materiales compuestos, gracias a su capacidad de modelado de diferentes geometrías complejas y el análisis del efecto de ellas sobre el material resultante. En el caso específico de partículas, éstas han sido modeladas exitosamente utilizando tanto modelos 2D como 3D, mediante figuras geométricas tales como cubos, cilindros, cilindros truncados, conos, conos truncados, doble conos y esferas [6]. Habitualmente los resultados obtenidos se comparan con los modelos teóricos anteriormente mencionados y con mediciones experimentales, con la finalidad de validar el modelo usado. Gracias a las facilidades de cómputo en cuanto a geometría, modelación y simulación, problemas tridimensionales complejos se han tratado como bidimensionales utilizando aproximaciones axi-simétricas, así como de esfuerzo o deformación planos. Es difícil modelar la microestructura real del compuesto, ya que en la mayoría de los casos presentan distribuciones aleatorias. Por tal motivo habitualmente se asumen celdas unitarias con arreglos periódicos, que pueden ser cuadrados, circulares o hexagonales (cúbicos o cilíndricos en 3D). Algunos ejemplos de estos trabajos se analizarán a continuación, utilizando diferentes modelos, celdas unitarias y elementos volumétricos representativos (RVE), pudiendo ser modelos de varias partículas o de una sola para optimizar las capacidades de cómputo [ㅁ, 29].

Un ejemplo del estudio del comportamiento mecánico de compósitos mediante el MEF, con apoyo computacional, es el trabajo de Devireddy y Biswas [30], que corresponde a un material compuesto de matriz 
polimérica reforzado con fibras de vidrio unidireccionales, analizándose el comportamiento mecánico y térmico en dependencia de la fracción volumétrica del reforzante. La matriz constitutiva anisotrópica utilizada para el análisis corresponde a un material homogéneo y elástico, dada por la Ley de Hooke como:

$$
\left\{\begin{array}{l}
\sigma_{1} \\
\sigma_{2} \\
\sigma_{3} \\
\sigma_{4} \\
\sigma_{5} \\
\sigma_{6}
\end{array}\right\} \equiv\left\{\begin{array}{l}
\sigma_{x x} \\
\sigma_{y y} \\
\sigma_{z z} \\
\tau_{y z} \\
\tau_{z x} \\
\tau_{x y}
\end{array}\right\}=\left[\begin{array}{llllll}
C_{11} & C_{12} & C_{13} & C_{14} & C_{15} & C_{16} \\
C_{12} & C_{22} & C_{23} & C_{24} & C_{25} & C_{26} \\
C_{13} & C_{23} & C_{33} & C_{34} & C_{35} & C_{36} \\
C_{14} & C_{24} & C_{34} & C_{44} & C_{45} & C_{46} \\
C_{15} & C_{25} & C_{35} & C_{45} & C_{55} & C_{56} \\
C_{16} & C_{26} & C_{36} & C_{46} & C_{56} & C_{66}
\end{array}\right]\left\{\begin{array}{l}
\varepsilon_{x x} \\
\varepsilon_{y y} \\
\varepsilon_{z z} \\
\gamma_{y z} \\
\gamma_{z x} \\
\gamma_{x y}
\end{array}\right\} \equiv\left\{\begin{array}{l}
\varepsilon_{1} \\
\varepsilon_{2} \\
\varepsilon_{3} \\
\varepsilon_{4} \\
\varepsilon_{5} \\
\varepsilon_{6}
\end{array}\right\}
$$

donde $\sigma_{i j}$ y $\tau_{i j}$ son respectivamente los componentes normal y de corte del esfuerzo, $\varepsilon_{i j}$ y $\gamma_{i j}$ los componentes normales de la deformación, y $C_{i j}$ la matriz simétrica de rigidez, con 21 constantes elásticas independientes. El material compuesto en este caso se considera como transversalmente isotrópico, por lo que es posible calcular los módulos de rigidez longitudinal y transversal mediante las ecuaciones (2) y (3), respectivamente:

$$
\begin{gathered}
E_{1}=C_{11}-\frac{2 C_{12}^{2}}{\left(C_{22}+C_{23}\right)}, \\
E_{2}=\frac{\left[C_{11}\left(C_{22}+C_{23}\right)-2 C_{12}^{2}\right]\left(C_{22}-C_{23}\right)}{\left(C_{11} C_{22}-C_{12}^{2}\right)}
\end{gathered}
$$

En este trabajo utilizan diferentes arreglos de fibras, como puede apreciarse en la Figura 2, demostrando lo anteriormente comentado sobre la validez del uso de diferentes celdas unitarias y RVE. Para generar cierto RVE es importante tomar en cuenta la fracción volumétrica del reforzante dentro del compuesto, y a su vez las formas del reforzante y del RVE, para poder realizar los cálculos correctos. Asimismo es importante el planteamiento del modelo y sus simplificaciones, tales como son el tipo de arreglo de la fibra, la ausencia de irregularidades, la distribución homogénea, la alineación perfecta de las fibras y el tipo de unión en la interfase. También es de vital importancia la selección correcta de las condiciones de frontera, cargas, desplazamientos, etc. En casos como el que se muestra en este trabajo se puede utilizar un modelo bidimensional, lo cual es posible debido al comportamiento del modelo dentro del estado de deformación plana.
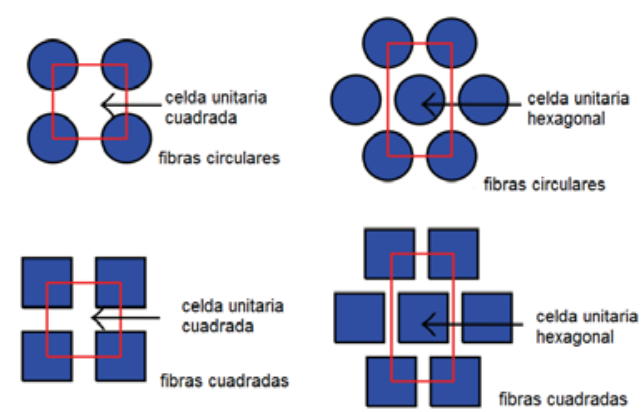

Figura 2: Arreglos de fibras continuas para el modelado de materiales compuestos, utilizando secciones transversales redondas y cuadradas, seleccionando diferentes celdas unitarias. Modificado de [30].

En este caso se analizó el efecto de la fracción volumétrica de las fibras sobre el módulo de Young y sobre la distribución de los esfuerzos generados en el material. En la Figura 3a se observa el mallado con elementos sólidos y los resultados de la distribución de esfuerzos (Figura 3b) y deformaciones (Figura 3c). Existen algunos trabajos similares a este, entre los cuales está el de Pal y Haseebuddin [31], que estudia el efecto del contenido de fibras reforzantes sobre el módulo de Young; o el de Joshi y col.[32], que analiza el efecto del tipo de fibra. De igual forma se ha utilizado este método para analizar la influencia de grietas y la desunión reforzante-matriz [33-36], que puede modificarse de acuerdo a la fortaleza de la unión. Incluso el MEF permite el modelado de interfases, muy útil para el estudio de sistemas en los que ocurren reacciones, modelando estas interfases como un tercer material $[\underline{37}, \underline{38}]$. Sobre este tema se observó que sería importante un mayor estudio. 

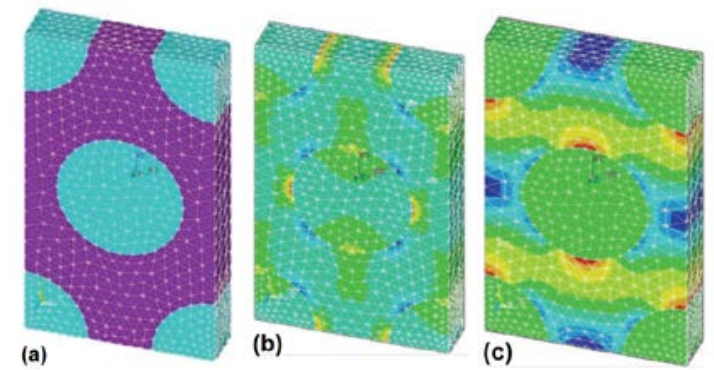

Figura 3: Resultados típicos para: (a) Mallado del modelo de fibras con secciones transversales circulares, ordenadas de forma hexagonal; y los resultados obtenidos para la distribución de: (b) esfuerzos y (c) deformaciones. Modificado de [30].

En la Figura 4a-b, se puede observar la gráfica comparativa entre los resultados obtenidos para el módulo de Young de acuerdo a la fracción volumétrica de reforzantes para los modelos de la Figura 2. Es de destacar que para los módulos transversales los resultados son muy variados (Figura 4b). Esta comparación se realiza con la finalidad de mostrar la eficacia del MEF, así como el efecto de la celda unitaria o el tipo de fibra.

(a)
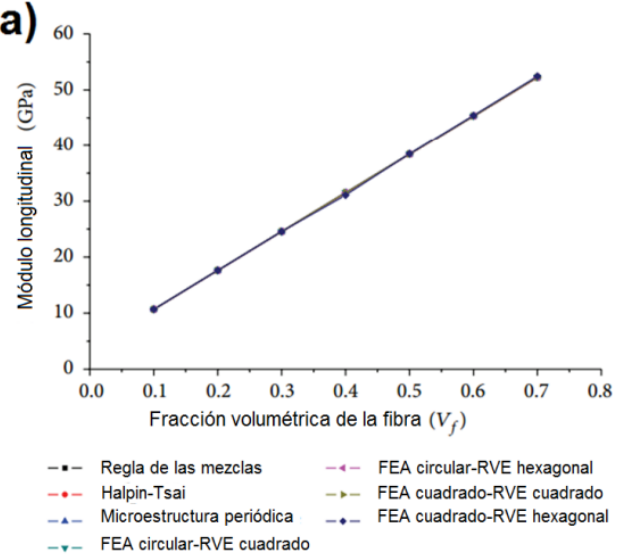

(b)

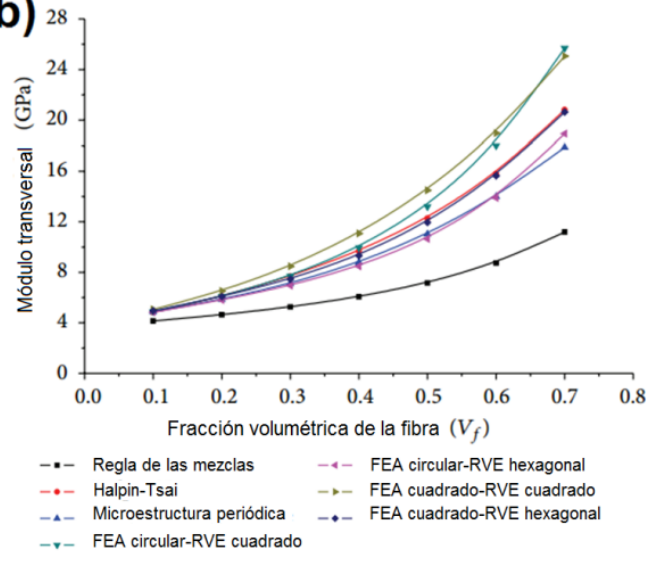

Figura 4: Módulos de Young longitudinales (a) y transversales (b) de acuerdo a la fracción volumétrica de reforzantes,

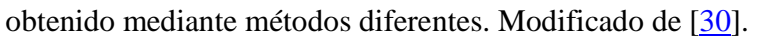

Utilizando elementos finitos también es posible simular materiales mediante el análisis de micrografías en lugar de realizar modelaciones, ya sea a partir de imágenes 2D [] ] o 3D, como las obtenidas mediante tomografía de rayos-X [풍. Tal es el caso de un material compuesto de matriz de aluminio reforzado con partículas de SiC, lo cual puede observarse en la Figura 5a, donde se utilizan elementos triangulares para conformar la estructura real. En este caso, se obtienen micrografías de la estructura y se replican, para así conformar el modelo y realizar la simulación [ㅁ] . Esta figura muestra el especial cuidado que se debe tener en la unión matriz-reforzante, que es la zona crítica. Al estar constituido por dos materiales con propiedades mecánicas diferentes, el mallado debe indicar esta discontinuidad. En este trabajo se estudia el efecto de la orientación y la fracción volumétrica de las partículas sobre los módulos de Young longitudinal y transversal, así como sobre los esfuerzos generados, y se comparan de manera sobresaliente (ver Figura 5b) con los resultados experimentales y con otros modelos teóricos, como el de Halpin-Tsai y de Hashin-Shtrikman. 

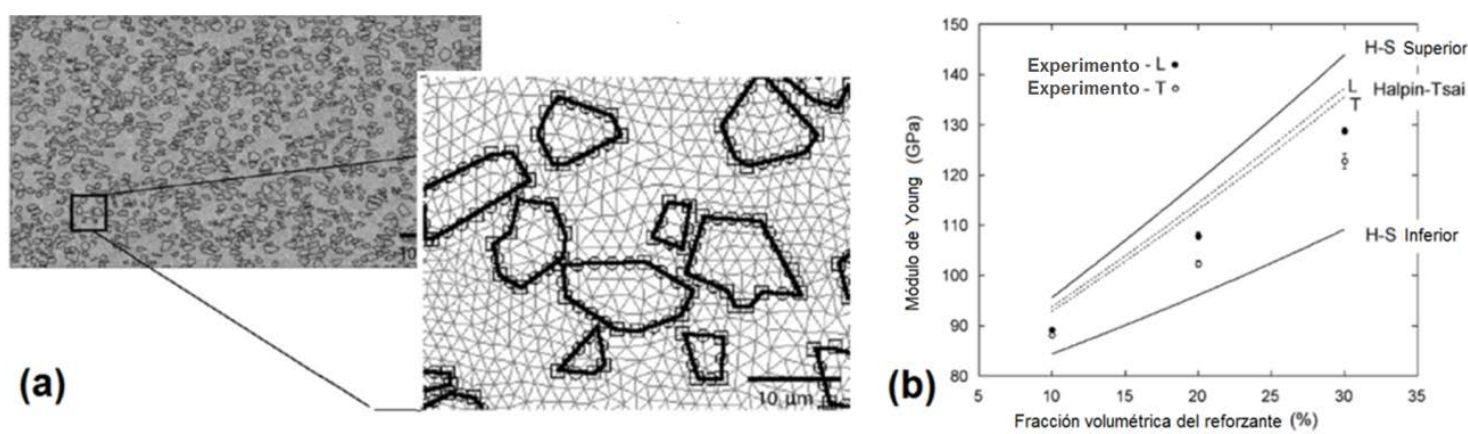

Figura 5: (a) Microestructura de un material compuesto de matriz metálica obtenida mediante la réplica de una imagen de Microscopía Electrónica de Barrido y su modelación. (b) Estimación del Módulo de Young de acuerdo a la fracción volumétrica del reforzante. Modificado de []].

En muchos casos la modelación de los materiales compuestos se puede realizar tanto en 2-D como en $3-\mathrm{D}$, gracias a las facilidades que nos ofrecen los programas computacionales. Tal es el caso del uso de modelos axi-simétricos. No obstante, se ha encontrado que sus resultados son diferentes debido a que cambia el tamaño del RVE, siendo solo cilíndrico en el caso del modelo axi-simétrico, mientras que en el modelo tridimensional puede ser cilíndrico, prismático o hexagonal $[\underline{19}, \underline{40}, \underline{41}]$. Un ejemplo de lo anterior es la estimación del módulo de Young en dependencia de la relación de aspecto y la fracción volumétrica, para un material compuesto reforzado con partículas cilíndricas [41]. En estos modelos las relaciones diámetro-altura para el modelo 2D difieren notablemente de la relación base-altura para el modelo 3D, originando que cambie el comportamiento mecánico del compuesto. Para estos modelos, las condiciones de frontera para el modelo axi-simétrico son:

$$
U_{Z}\left(R, L_{Z}\right)=U ; \quad U_{R}(0, z)=0 ; \quad U_{Z}(x, 0)=0
$$

mientras que para el modelo 3D son:

$$
U_{Z}\left(x, y, L_{Z}\right)=U ; \quad U_{X}(0, y, z)=0 ; \quad U_{Y}(x, 0, z)=0 ; \quad U_{Z}(x, y, 0)=0
$$

En la Figura 6a-c pueden observarse los modelos 3D propuestos para partículas con relaciones de aspecto de 0.2 , 1.0 y 1.8. Este modelo utiliza solo una octava parte del sistema partícula-matriz, y elementos sólidos (solid). En el caso de los modelos 2D se utilizan elementos planos (plane) con la opción de axisimetría. En la Figura 6d-f se muestra el comportamiento de los esfuerzos normales para cada caso, ubicados los máximos (de color rojo) en la interfase partícula-matriz, donde generalmente inician fallas en estos materiales.
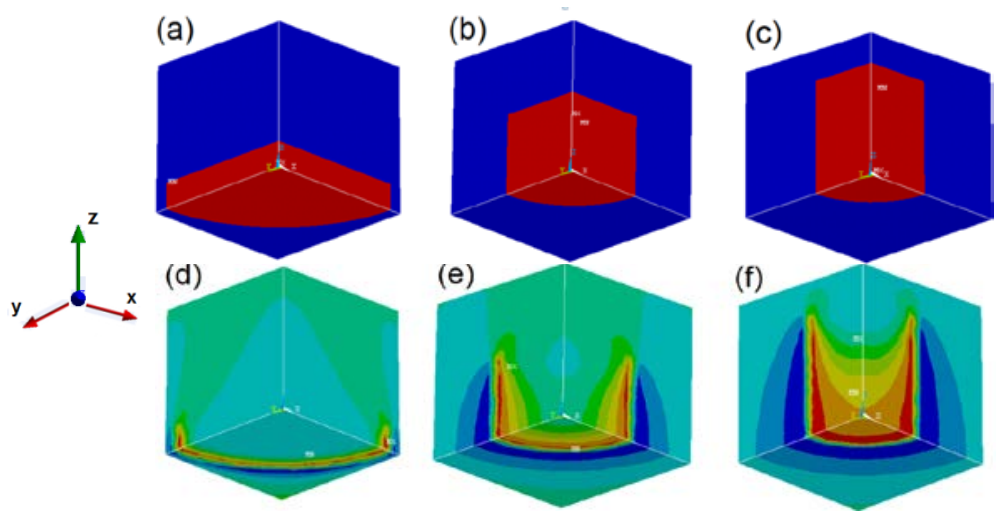

Figura 6: (a-c) Modelado mediante el MEF de la celda partícula-matriz para partículas cilíndricas con diferentes relaciones de aspecto. (d-f) Distribución de los esfuerzos normales. Modificado de [1]].

En este tipo de modelos las restricciones de movimiento y el tipo de unión reforzante-matriz también juegan un papel fundamental, ya que es necesario restringirse el movimiento de los nodos del fondo, mientras que los nodos de los planos YZ y XZ fueron restringidos en las direcciones X y Y, respectivamente. En la Figura 7a, se puede observar la gráfica comparativa para los valores del módulo de Young, obtenidos por el modelo de MEF mostrado en la Figura 6, por el modelo de Halpin-Tsai y por la regla de las mezclas, en to- 
dos los casos relacionado con la relación de aspecto del reforzante. Se aprecia que los resultados obtenidos mediante FEM están en el rango de los que predicen el modelo de Halpin-Tsai y la regla de las mezclas, mostrando la eficacia del modelo empleado. Los valores estimado por el MEF 3D fueron los más elevados y a su vez los más cercanos a las mediciones experimentales. En este trabajo también se observaron las diferencias significativas que se obtienen al utilizar modelos 2D y 3D, coincidiendo con lo reportado por Saraev y Shamauder [40], ilustrado en la Figura 7b.
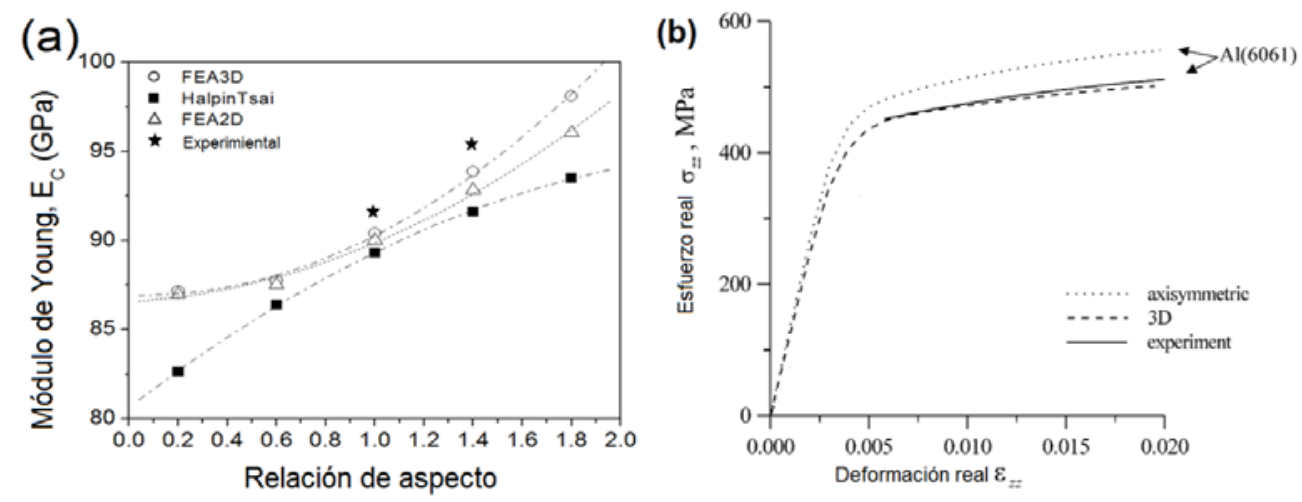

Figura 7: (a) Efecto de la relación de aspecto sobre el Módulo de Young para un material compuesto de matriz de aluminio reforzado con partículas de SiC modificado de [41]. (b) Curvas esfuerzo-deformación para un material compuesto dependiendo del tipo de modelo usado, 2D ó 3D. Modificado de [누].

Otros trabajos estudian a los materiales compuestos mediante el MEF, analizando la influencia de la orientación del reforzante []], como puede observarse en la Figura 8 a-c, donde se modelan materiales compuestos con fibras cortas ordenadas aleatoriamente. En este caso, pueden observarse los elementos sólidos (solid) utilizados en el mallado. Los resultados obtenidos permiten estimar parámetros como el módulo de Young, relacionándolo con la orientación de los reforzantes y su fracción volumétrica. Este tipo de trabajo también se ha llevado a cabo utilizando diferentes modelos de partículas o fibras cortas [42, 43], siendo modelos tridimensionales que emplean un gran número de partículas, representando dificultades de modelación y cómputo mayores, al contrario de otros modelos anteriormente analizados donde la celda unitaria solo emplea una partícula o parte de ellas. En la presente revisión se observó que el estudio de los reforzantes en direcciones preferenciales fue más realizado que en direcciones aleatorias.
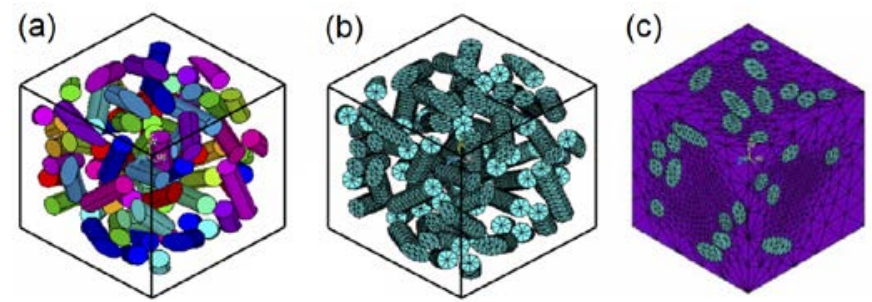

Figura 8: Modelación de un material compuesto reforzado mediante fibras cortas sin orientación preferencial. Se puede observar el ordenamiento (a), el mallado de las fibras (b) y del material (c). Modificado de [7].

Al estudio que se ha venido realizando utilizando MEF en materiales compuestos reforzados por partículas y fibras, se ha sumado el análisis de nanotubos de carbono [44-46]. En la Figura 9a, se aprecia un ejemplo del modelado de nanotubos de carbono, donde cada enlace C-C se toma como un elemento tipo "beam", mientras que los átomos de carbono se modelan como nodos. En estos modelos la energía total de enlace se representa como la energía de deformación total de una viga uniforme de longitud $l$ y sección transversal $A$. En la Figura 9b, se puede observar el modelado de un nanotubo, destacándose las condiciones de carga y restricciones de movimiento, mientras que en la Figura 9c se muestra cómo se inserta el nanotubo de carbono en el modelo de un material compuesto. Sobre este tema, se encontraron otros trabajos, como el de Reddy y col. [17], o el de Davoudabadi y col. [47], quienes estudiaron con éxito el efecto de la relación de aspecto del nanotubo de carbono sobre el módulo de Young, comparándolo con la regla de las mezclas y el modelo de Halpin-Tsai para un material compuesto de matriz polimérica. Otros trabajos estudian las interfases y los problemas surgidos por falta de unión [뇨, $\underline{49}]$. 
(a)

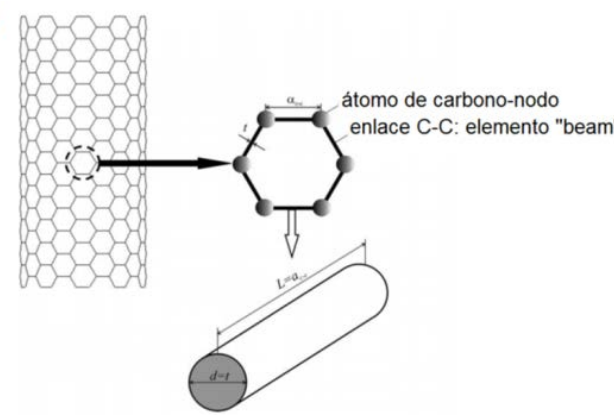

(b)

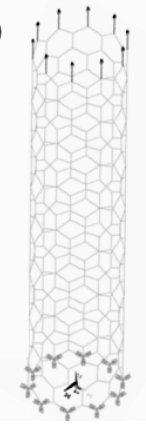

(c)

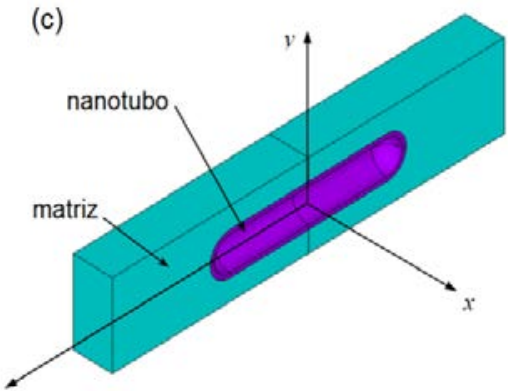

Figura 9: Modelación de un material compuesto reforzado por nanotubos de carbono: (a) modelo, (b) representación computacional del nanotubo de carbono, y (c) representación de la matriz y el nanotubo de carbono. Modificado de [녀46]

\section{CONCLUSIONES}

Derivado del análisis bibliográfico realizado en el presente trabajo se puede concluir lo siguiente:

1. El Método de los Elementos Finitos (MEF) es una herramienta muy eficiente para el estudio de materiales compuestos, posibilitando estimar su comportamiento mecánico. Se notó que la propiedad más estudiada es el módulo de Young. El estudio de otras propiedades como la relación de Poisson deberían ser incluidas.

2. Los efectos más estudiados son la fracción volumétrica y la relación de aspecto de los reforzantes. Se observó que el estudio de los reforzantes en direcciones preferenciales fue más realizado que en direcciones aleatorias.

3. Se han reportado un gran número de modelos para generar la estructura de diferentes materiales compuestos, tomando en cuenta diferentes sistemas que contienen el sistema matriz-reforzante. Se observó que muchos de estos modelos utilizan las posibilidades de simplificación geométrica que ofrecen los programas de cómputo, de acuerdo a la simetría de los materiales, siendo factible la optimización. El uso de celdas unitarias o RVE se ve favorecido por su sencillez.

4. No se encontraron suficientes trabajos que realicen el modelado a partir de microestructuras obtenidas por análisis de imágenes. Igualmente se encontraron insuficientes trabajos que aborden la formación de interfases.

\section{AGRADECIMIENTOS}

Los autores agradecen el apoyo financiero de los proyectos UNAM PAPITT TA100114 y SENER CONACYT 151496, para el desarrollo del presente trabajo.

\section{BIBLIOGRAFÍA}

[1] ZIENKIEWICZ, O.C., TAYLOR, R.L., El Método de los Elementos Finitos. Volumen 1. Formulación básica y problemas lineales, 4 ed., McGraw-Hill, 1994.

[2] BELTRÁN, F., Teoría General del Método de los Elementos Finitos. Curso de Doctorado. Departamento de Mecánica Estructural y Construcciones Industriales - ETS Ingenieros Industriales. Madrid, 1999.

[3] TURNER, M.J., CLOUGH, R.W., MARTIN, H.C., Y TOPP, L.J., "Stiffness and deflection analysis of complex structures”, Journal of the Aeronautical Sciences, v. 23, n. 9, pp. 805-824, Sept.1956.

[4] STRANG, G., FIX, G.J., An Analysis of the Finite Element Method, Prentice-Hall, 1973.

[5] JUNG, H.K., CHEONG, Y.M., RYU, H.J., et al., “Analysis of anisotropy in elastic constants of SiCp/2124 al metal matrix composites”, Scripta Materialia, v. 41, n. 12, pp. 1261-1267, Nov.1999.

[6] GANESH, V.V., CHAWLA, N., "Effect of particle orientation anisotropy on the tensile behavior of metal matrix composites: experiments and microstructure-based simulation”, Materials Science and Engineering A, v. 391, pp. 342-353, Jan. 2005. 
[7] KARI, S., BERGER, H., GABBERT, U., "Numerical evaluation of effective material properties of randomly distributed short cylindrical fibre composites”, Computational Materials Science, v. 39, n. 1, pp. 198204, Mar. 2007.

[8] MOHAMMADPOUR, E., AWANG M., "Predicting the nonlinear tensile behavior of carbon nanotubes using finite element simulation”, Applied Physics A: Materials Science \& Processing, v.104, n. 2, pp. 609614, Aug. 2011.

[9] CADENA, J.H., ALFONSO, I., RAMIREZ, J.H., et al., "Improvement of FEA estimations for compression behavior of Mg foams based on experimental observations", Computational Materials Science, v. 91, pp. 359-363, 2014.

[10] PARIONA, M. M., MOSSI, A. C., "Numerical simulation of heat transfer during the solidification of pure iron in sand and mullite molds", Journal of the Brazilian Society of Mechanical Sciences and Engineering, v. 27, n. 4, pp. 399-406, Oct. 2005.

[11] IGNASZAK, Z., HAJKOWSKI, M., HAJKOWSKI, J., "Prediction of dendritic microstructure using the cellular automaton-Finite Element Method for hypoeutectic Al-Si Alloys Castings", Materials Science (Medžiagotyra), v. 12, n. 2, pp. 124-128, Apr. 2006.

[12] BAOQING, L., SHAN, J., LIANI, S., et al., "Finite Element Simulation of the process of aluminum alloy resistance spot welding”, In: Proc. 20th CAD-FEM Users Meeting, International Congress on FEM Technology. Friedrichshafen, Germany, Oct. 2002.

[13] PAIK, J.P., LEE, J.M., KO, M.J., "Ultimate compressive strength of plate elements with pit corrosion wastage”, In: Proc. of the Institute of Mechanical Engineers. v. 217, Part M: Engineering for Maritime Enviroment, pp. 185-200, Dec. 2003.

[14] ARAUJO, R., DIAS, A.M.S., GODOY, G.C.D., "Numerical study of the influence of friction coefficient for indentation testing in thin films", Matéria, v.18, n.1, pp. 55-66, 2013.

[15] ANSYS 15.0 M.R. Help.

[16] ENGINEERING Materials Handbook. Volume 1: Composites. ASM International, 3 ed., 1989.

[17] CHAWLA, N., CHAWLA, K.K., Metal Matrix Composites, Kluwer Academic Publishers, 2004.

[18] RAMGOPAL REDDY, B., RAMJI, K., SATYANARAYANA, B., "Effect of fiber orientation on elastic properties of CNT based glass/epoxy lamina-FEA approach”, International Journal of Nanotechnology and Application, v. 4, n. 2, pp. 153-162, May. 2010.

[19] CHAWLA, N., SHEN, Y.L., "Mechanical behavior of particle reinforced metal matrix composites", Advanced Engineering Materials, v. 3, n. 6, pp. 357-370, 2001.

[20] KUMAR, A.P., DEPAN, D., TOMER, N.S., SINGH, R.P., "Nanoscale particles for polymer degradation and stabilization-Trends and future perspectives", Progress in Polymer Science, v. 34, pp. 479-515, 2009. [21] HALPIN, J.C., KARDOS, J.L., "The Halpin-Tsai Equations: A Review". Polymer engineering and science, v. 16, n. 5, pp. 344-352, May. 1976.

[22] HASHIN, Z., SHTRIKMAN, S. "A variational approach to the theory of the elastic behaviour of multiphase materials", Journal of the Mechanics and Physics of Solids, v. 11, n. 2, pp.127-140, 1963.

[23] ESHELBY, J.D., "The determination of the elastic field of an elliptical inclusion and related problems", Proc. R. Soc. Lond., A241, pp. 376-396, 1957.

[24] MURA, T., Micromechanics of Defects in Solids, Martinus Nijhoff, 2 ed., The Hague, 1987.

[25] KRONER, E., "Calculation of the elastic constant of a polycrystal from the constant of a single crystal", Z. Phys., v. 151, pp. 504-518, 1958.

[26] BUDIANSKY, B., "On the elastic moduli of some heterogeneous materials", Journal of the Mechanics and Physics of Solids, v. 13, pp. 223-227, 1965.

[27] GUTH, E., "Theory of filler reinforcement", Journal Applied Physics, v.16, pp. 20-25, 1945.

[28] LEWIS. T.B., NIELSEN. L.E., "Dynamic mechanical properties of particulate-filled composites", Journal of Applied Polymer Science, v. 14, n. 6, 1449-1471, 1970. 
[29] PAHLAVANPOUR, M., MOUSSADDY H. H., GHOSSEIN, E., et al., "Prediction of elastic properties in polymer-clay nanocomposites: Analytical homogenization methods and 3D finite element modeling", Computational Materials Science, v. 79, pp. 206-215, 2013.

[30] SIVA BHASKARA RAO DEVIREDDY, SANDHYARANI BISWAS "Effect of fiber geometry and representative volume element on elastic and thermal properties of unidirectional fiber-reinforced composites", Journal of Composites, v. 2014, 12 pages.

[31] PAL, B., HASEEBUDDIN, MR., "Analytical estimation of elastic properties of polypropylene fiber matrix composite by Finite Element Analysis", Advances in Materials Physics and Chemistry, v. 2, pp. 23-30, 2012.

[32] JOSHI, U.A., JOSHI, P., HARSHA, S. P., SHARMA, S.C., "Evaluation of the mechanical properties of carbon nanotube based composites by Finite Element Analysis", International Journal of Engineering Science and Technology, v. 2, n. 5, pp. 1098-1107, 2010.

[33] JOHNSON, A.C., ZHAO, F.M., HAYES, S.A., et al., "Influence of a matrix crack on stress transfer to an $\alpha$-alumina fibre in epoxy resin using FEA and photoelasticity", Composites Science and Technology, v. 66, n. 13 pp. 2023-2029, Oct. 2006.

[34] THIONNET, A., RENARD, J., "Multi-scale analysis to determine fibre/matrix debonding criteria in SiC Titanium composites with and without consideration of the manufacturing residual stresses", Composites Science and Technology, v. 58, n. 6, pp. 945-955, 1998.

[35] ZHANG, X., LIU, H., MAI, Y., "Effects of fibre debonding and sliding on the fracture behaviour of fibre-reinforced composites”, Composites. Part. A-Appl. S., v. 35, n. 11, pp. 1313-1323, Nov. 2004.

[36] SRIHARI, P.A., RAMANA, K.V., BALAKRISHNA, MURTHY V., et al., "Role of finite element method (FEM) in predicting transverse modulus of fiber-reinforced polymer (FRP) composites: A revelation". International Journal of Physical Sciences, n. 8, v.29, pp. 1526-1536, 9 August, 2013.

[37] ZHANG, W.X., Li, L.X., WANG, T.J., "Interphase effect on the strengthening behavior of particlereinforced metal matrix composites", Computational Materials Science, v. 41, n. 2, pp. 145-155, 2007.

[38] DAIBIN GE, MINGYUAN GU, "Mechanical properties of hybrid reinforced aluminum based composites", Materials Letters, v. 49, pp. 334-339, 2010.

[39] CROSTACKA, H.A., NELLESENB, J., FISCHERB, G., et al., "Tomographic Analysis and FESimulations of MMC-Microstructures under Load", Proc. of SPIE, v. 6318, 63181A, 2006.

[40] SARAEV, D., SCHMAUDER, S., "Finite element modelling of Al/SiCp metal matrix composites with particles aligned in stripes a 2D-3D comparison", International Journal of Plasticity, v. 19, pp.733-747, 2003.

[41] ALFONSO, I., FLORES, J.E., FIGUEROA, I., et al., "Young's modulus estimation of a MMC with different particle aspect ratios", In: XX International Materials Research Congress. Symposium 2. Advances in Computational Materials Science, Cancún, México, 14-19 Aug. 2011.

[42] SRIVASTAVA, V. K., GABBERT, U., BERGER, H., et al., "Analysis of particles loaded fiber composites for the evaluation of effective material properties with the variation of shape and size", International Journal of Engineering, Science And Technology, v. 3, n. 1, pp. 52-68, 2011.

[43] SHEN, H., LISSENDEN, C.J., "Stress and strain localization three-dimensional modeling for particlereinforced Metal Matrix Composites", Metallurgical and Materials Transactions A, v. 36A, pp. 1653-1660, 2005.

[44] FAN, C., HUANG, J., HWU, C., LIU, Y., “A Finite Element approach for estimation of Young’s Modulus of single-walled carbon nanotubes", In: The Third Taiwan-Japan Workshop on Mechanical and Aerospace Engineering. Hualian, Taiwan, R.O.C. 28-29 Nov. 2005.

[45] JALALAHMADI, B., NAGHDABADI, R., "Finite element modeling of single-walled carbon nanotubes with introducing a new wall thickness", In: Proceedings of the International Conference on Nanoscience and Technology (ICN\&T, Basel, Switzerland, Jul. 2006), Journal of Physics Conference Series, v. 61, pp. 497502, 2007. 
[46] LIU, Y. J., CHEN, X. L., “Continuum models of carbon nanotube-based composites”, Electronic Journal of Boundary Elements, v. 1, n. 2, pp. 316-335, 2003.

[47] DAVOUDABADI, M.R., EBRAHIMI NAGHANI, R., FEREIDOON, A., "Prediction of Young's Modulus of carbon nanotube reinforced composites in axial direction with nonlinear springs", World Applied Sciences Journal, v. 10, n. 9, pp. 1007-1019, 2010.

[48] SAFFAR, K., JAMILPOUR, N., NAJAFI, A., et al., "A Finite Element Model for estimating Young’s Modulus of Carbon nanotube reinforced composites incorporating elastic cross-links”, International Journal of Aerospace and Mechanical Engineering, v. 2, n. 3, 172-175, Summer 2008.

[49] NEEDLEMAN, A., BORDERS, T.L., BRINSON, L.C., FLORES, V.M., SCHADLE, L.S., "Effect of an interphase region on debonding of a CNT reinforced polymer composite", Composites Science and Technology, v. 70, n. 15, pp. 2207-2215, Dec. 2010. 